\title{
Understanding formability and geometrical accuracy of SPIF process used as Reshaping approach
}

\author{
Omer Zaheer, Giuseppe Ingarao, Rosa Di Lorenzo and Livan Fratini
}

\author{
Omer Zaheer. Department of Engineering, University of Palermo, Viale delle Scienze, Palermo, 90128, Italy \\ Corresponding author: Omer Zaheer. E-mail address: omer.zaheer@community.unipa.it \\ Giuseppe Ingarao. Department of Engineering, University of Palermo, Viale delle Scienze, Palermo, 90128, Italy \\ Rosa Di Lorenzo. Department of Engineering, University of Palermo, Viale delle Scienze, Palermo, 90128, Italy \\ Livan Fratini. Department of Engineering, University of Palermo, Viale delle Scienze, Palermo, 90128, Italy
}

\begin{abstract}
Putting in place Circular Economy strategies is an urgent action to be undertaken. Manufacturing processes play a relevant role as efficient material reuse enabler. Scientists have to make an effort either to find new process or to rethink old process to reprocess End-of-life (EoL) components to recover both material and functions. In this paper, Single Point Incremental Forming (SPIF) process is used for reshaping sheet metal EoL components. Deep drawing process as well as uniaxial pre-straining (to imitate the End-of-Life component) followed by SPIF operations (to obtain the reshaped components) are set- up and implemented to form and reform aluminum sheet metal components. As the authors have already proved the technical feasibility of such an approach, the present paper aims at a better understanding of the formability and geometrical accuracy performance of SPIF process as used to reform components. Specifically, an experimental campaign varying kind and extent of restraining is developed and the formability and geometrical accuracy of the subsequent SIPF operations is analyzed. Results proves that SPIF process is a promising approach for reshaping purpose.
\end{abstract}

Keywords. Circular Economy, Reshaping, SPIF, Formability

\section{Introduction}

Reducing anthropogenic environmental impact is an urgent issue to deal with. One of the main contributors to yearly CO2 emissions is materials production, accounting for about 25\% of the global CO2 emissions [1]. Reduction of the environmental impact of material production could be achieved by the implementation of a Circular Economy paradigm. Intense usage, product repair and upgradation, remanufacturing, component re-use and open/closed loop recycling are some of the strategies that could contribute towards reducing the environmental impact of raw material production [2]. The main principle focuses on turning an EoL product/component directly either into a reusable material or, better yet, into new products/components. As far as metals are concerned, recycling is still the most applied strategy as it provides environmental, technological and economic benefits. Nevertheless, it is, by now, urgent turning to more virtuous circular economy strategies, such us product/component reuse. Besides material itself, reuse strategies would also allow functions recovery from EoL components. In this scenario manufacturing scientists play a relevant role as they are called to think of new processes or to rethink conventional processes to be applied as circular economy enabler. As far as metals EoL components are concerned, a "Reuse" framework was proposed by Cooper and Allwood [3]. To be more specific, in this framework four main strategies are identified; two of them rely on superficial reconditioning and the product/component are reused either for the same type of function (in the case of Relocate) or for a less demanding use (in case of Cascade). The other two envisage "The component(s) undergo extensive reconditioning" and these are: $1 /$ Remanufacturing, where inspection, disassembly, re-drilling, and metallic spraying/ thermal techniques are the process to be applied (typical remanufacturing applications for metals concern automotive engines and dies ); and 2/Reform/Reshape: where manufacturing approaches (additive, subtractive, mass conserving) are applied to obtain a new, more useful, geometry reprocessing the returned EoL component changing its shape. While Remanufacturing has been widely analyzed by manufacturing scientist over the last years [3] the Reshaping strategy has been overlooked by the scientific community so far. Looking specifically forming processes applied specifically as 
Understanding formability and geometrical accuracy of SPIF process used as Reshaping ap...

Reuse strategy for sheet metal components, very little scientific research has been developed so far. Brosius et al. [4] in a review paper describe how a demounted automotive engine-hood can be reshaped into a rectangular sheet metal component by sheet hydroforming process. Takano et al. [5] applied Single Point Incremental Forming (SPIF) on a flattened sheet. In fact, the Reshaping they propose includes the flattening of a previously bent sheet and a subsequent incremental forming step. Abu-Farha and Khraisheh [6] proposed the application of super plastic forming for applying Reshaping strategies on magnesium-based sheet components. These studies, although have the merit to propose the idea of Reshaping, they are preliminary ones and the potential of forming process in this new domain is not explored yet. The authors of the present paper have recently successfully applied Single Point Incremental forming SPIF to reshape sheet metal based EoL components [7]. Specifically, SPIF was used to change the shape of deep-drawn (DD) square box part. Along with the technical feasibility, the authors have proved the better energy efficiency with respect conventional and solid state recycling route [8]. Some issues characterizing such an approach are still to be analyzed, in fact there is a lack of process windows as well as of for process parameters influence analyses on formability accuracy. In this paper the change in formability and in the geometrical accuracy of SPIF when applied as Reshaping approach have been analyzed. Specifically, formability performance as well as geometrical accuracy with varying restraining types and level are analyzed. This paper, therefore, aims at analyzing such aspects and to provide guidelines to bring this approach closer to an industrial applicability.

\section{The proposed Reshaping approach}

The main aim of this research was to reshape previously deformed metal components in order to impart a different use. Such an approach would allow environmental impact reduction with respect conventional recycling route. The proposed approach relies on the possibility of recover large sheet metal components from EoL products and directly giving them a new shape through SPIF process. It is worth remarking that returned EoL components are normally characterized by high heterogeneity as there are localized thinning areas (caused by the original forming processes) while large part of the component underwent limited deformation. The thinned zones might be characterized by a limited formability while, in the less deformed zones it is still possible to take advantage of almost the entire original formability. Ideal manufacturing processes for reforming sheet metal based components should be characterized by higher formability as well as by a local action. this respect, Single Point Incremental Forming meets the aforementioned requirements perfectly. The mail idea of the proposed approach id sketched out in figure 1.

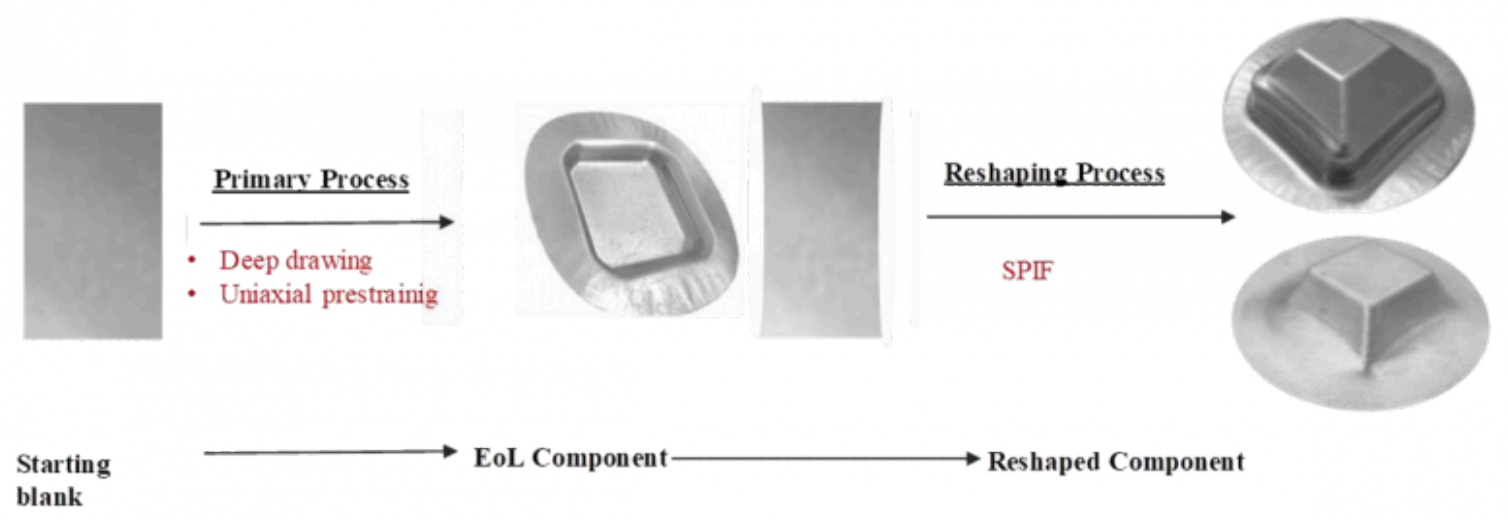

Fig. 1. The Proposed Reshaping approach.

In order to replicate the reshaping process chain, different Primary process and Reshaping ones were tested in the present research. Concerning the Primary process (the process to turn the virgin blank into the EoL components), 
two different processes were applied: deep drawing and uniaxial pre-straining. As far as the Reshaping process is considered, a truncated Pyramid was performed. For the case of the EoL component obtained by deep drawing, the SPIF process was applied on base of the square box along two different directions namely, Inward and Outwards.

\section{Materials and Methods}

For the experimental phase of the research, an AA5754 aluminum alloy was selected as the material to be studied. The experiments followed the selection of a $0.5 \mathrm{~mm}$ thick sheet. Three different kind of tests were performed, firstly the SPIF process was carried out on the virgin blank to form a truncated pyramid $15 \mathrm{~mm}$ in height and having a square base of $39 \mathrm{~mm}$. The main aim of first experimental campaign was to identify the maximum forming angle ( $\alpha$ max) of the incrementally formed pyramid. From now onwards $\alpha$ max will refer to the maximum forming angle at which no fracture of the part occurs. In order to do so, the flat base was changed by manufacturing a truncated pyramid with a base of $39 \mathrm{~mm}$ and $15 \mathrm{~mm}$ depth. In this research the clamping system for performing SPIF, was set up on a 4 axes CNC milling machine, a tool with a high-speed steel based $4 \mathrm{~mm}$ diameter was used. A helical tool path with a $0.3 \mathrm{~mm}$ descent was applied for each spire. Various tests were performed increasing the forming angle by half a degree each time a sane part was obtained. Three replications of each test were performed with the eventual identification of the maximum forming angle ( $\alpha$ max). Once, the maximum forming angle was determined, the research was focused on studying the influence of uniaxial pre-straining as well as Deep Drawing on the SPIF operation.

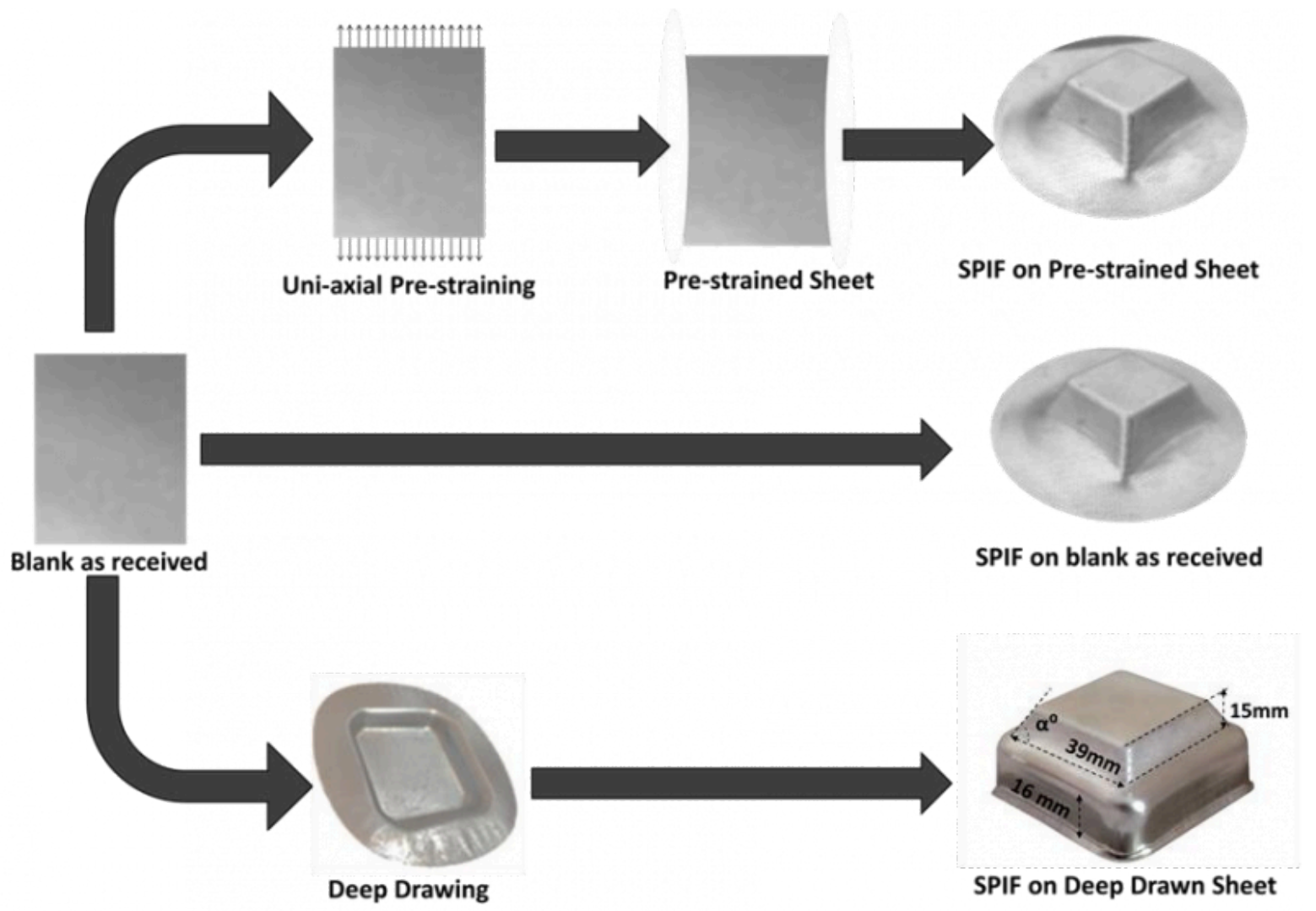

Fig. 2. Experimental campaign.

For the uniaxial pre-straining step, a virgin blank was subjected to two levels of uniaxial pre-straining, $15 \%$ and $10 \%$ of its original length. After the pre-straining step, a similar process was performed to determine the $\alpha$ max. Lastly, the 
Understanding formability and geometrical accuracy of SPIF process used as Reshaping ap...

effects of a deep drawing on the $\alpha$ max of a virgin blank were studied. For the deep drawing process, the blank was cut into a circular sheet, onto which a deep drawing process was performed to obtain a square cup $16 \mathrm{~mm}$ in height, having a side of $50 \mathrm{~mm}$ and a base fillet radius of $3 \mathrm{~mm}$. The flange from the deep drawing process was left in order to assist the clamping of the parts for SPIF. Similar to the first two cases, SPIF process was performed to identify the $\alpha$ max obtainable on the base of the square cup. Figure 2 depicts the three case studies carried out along with the geometrical dimensions of the parts.

The next step was the study of strain history of the critical area of the parts obtained at $\alpha$ max. For this, small circles $1.5 \mathrm{~mm}$ in diameter were laser incised on the samples. In the first case study of SPIF directly on a virgin blank, the change in the circles shape was recorded and used for the calculation of the occurring major and minor strains on the edge of the pyramid. In the cases of SPIF on pre-strained sheets, the strain history after the pre-straining steps was recorded along with the histories after the performing of SPIF process on at $\alpha$ max. Furthermore, with the aim to understand the effects of pre-straining process on the geometrical accuracy of SPIF process, some shape comparisons were developed. Specifically, the geometry obtained after the SPIF process compared to a reference CAD model of the pyramid. This comparison was developed for the $\alpha \max =64^{\circ}$ (the maximum obtainable in all implemented primary process) for all the four analyzed process chains. This was performed using a photo acquisition system, 'Steinbichler COMET' Laser Scanner. The acquired geometries were then analyzed through GeoMagic Control X software, which through the function of auto alignment between EoL and Reshaped part geometries, yielded the Maximum Deviation and the Root Mean Squared error. The obtained results from the experimental campaign are explained in detail in section 4.

\section{Results}

In figure 3 the obtained $\alpha$ max with varying the primary process is reported. As far as the reshaping samples with uniaxial prestraining are concerned, a slight decreasing trend is visible. Actually $\alpha$ max decreases by $1^{\circ}$ moving from the as received conditions up to the case with $15 \%$ of uniaxial pre-straining. These results prove that, although a decrease in formability is visible, the change is very limited and the SPIF process can still be successfully applied even after a significant amount of uniaxial pre-straining. Concerning the case with the DD used as primary process, no change in $\alpha$ max value was observed both in inward and in outwards case study. This can be explained considering that, in this case, the SPIF process is applied on zone characterized by a very limited (almost zero) degree of deformation, as a consequence the SPIF process can take advantage of the entire original material formability.

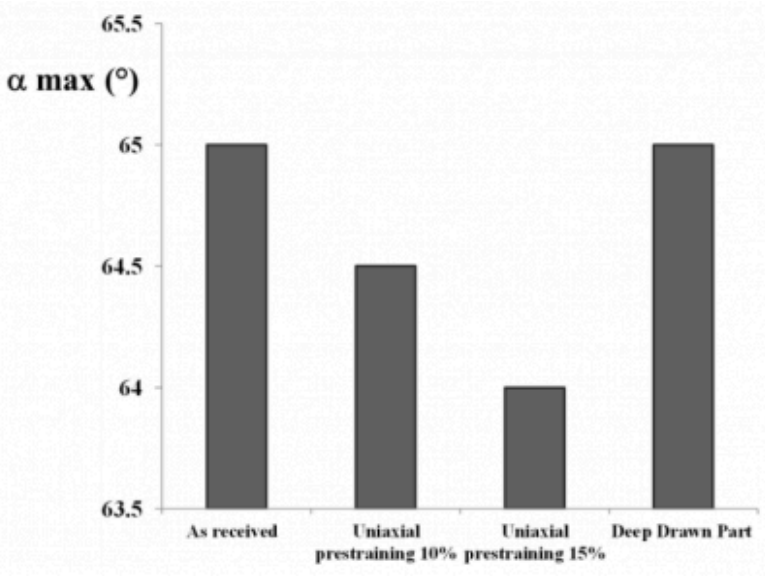

Fig. 3. Obtained $\alpha 仓$ max for the different primary processes considered 
In figure 4 the major strain-minor strain path some processes are reported. Specifically strain values measured on the edge of the SPIFed truncated pyramids are reported. For the sake of the clarity of representation only three paths are reported: pure SPIF ("As received" in figure), SPIF process applied on a flat sheet with $15 \%$ of uniaxial pre-straining and SPIF process applied on Deep drawn part. Again, the good amount of available formability is visible. The high values of major strain measured for both the reshaping approaches prove the potential of the proposed approach. The pre-straining of the uniaxial case is clearly visible and the strain path due to SPIF appears to be shifted leftwards. For the case of the Deep Drawing the two curves are almost the same because of the limited pre-straining values.

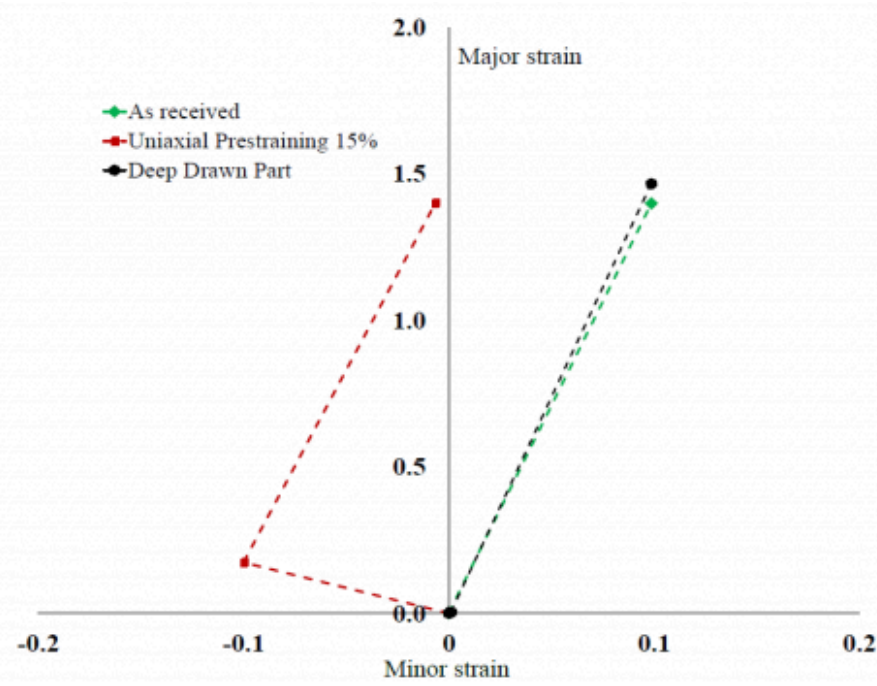

Fig. 4. $\alpha$ max Reshaping processes and pure SPIF Strain paths

As far as the numerical accuracy of SPIF used as a reshaping option is concerned, the results are summarized in table 1. In particular, a pyramid having an angle of $64^{\circ}$ was formed on the pre-strained and virgin blanks. The observed maximum deviation and the Root Mean Squared (RMS) error values are reported. It is possible to see that with the increase in the prestraining degree, the accuracy of the SPIF process improves.

Table 1. Deviation values of analyzed parts

\begin{tabular}{lcc}
\hline Part & Maximum Deviation (mm) & RMS \\
\hline Blank as received & 1.43 & 0.44 \\
\hline $15 \%$ uniaxial pre-strained blank & 1.16 & 0.23 \\
\hline Deep Drawn part & 1.34 & 0.38 \\
\hline
\end{tabular}

The results of the analysis are depicted in figure 5. For the sake of clarity only the deviation of the SPIFed area is analyzed, the error along the pyramid walls is visible on the three analyzed case studies. In any case, the higher rigidity of the pre-strained sheet reduces the rigid motion occurring due to SPIF. These early results prove the SPIF based Reshaping approach to be a promising one. 

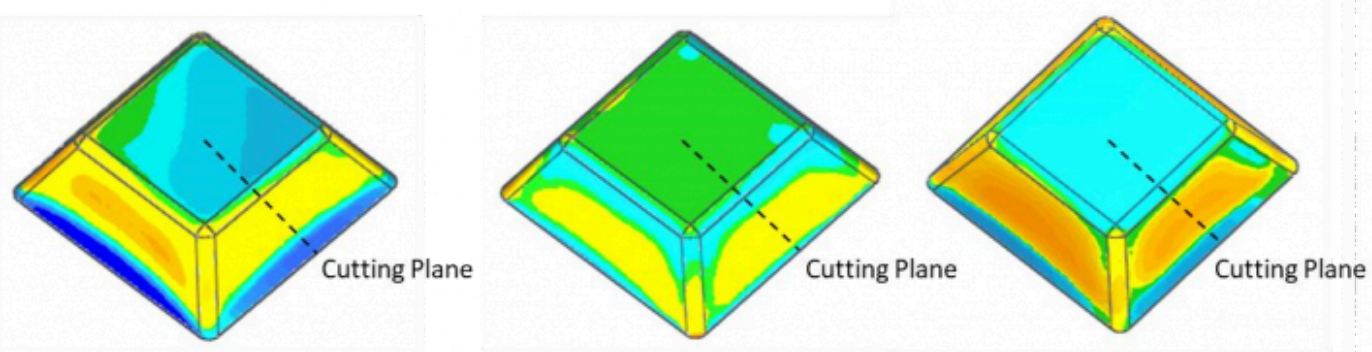

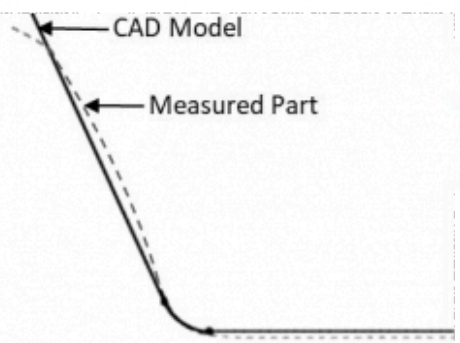

a)

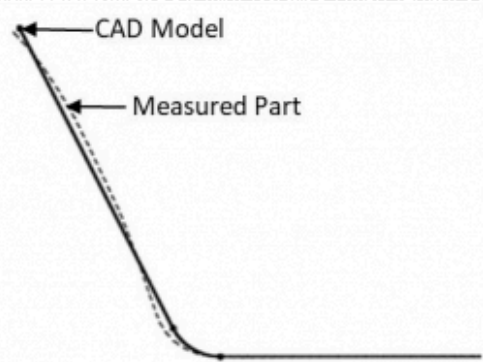

b)

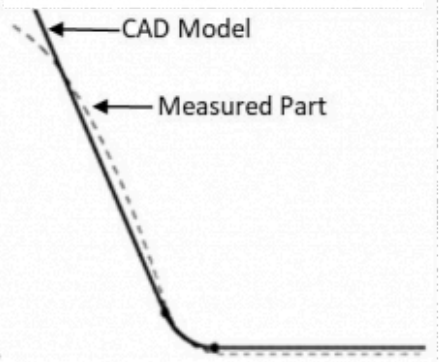

c)

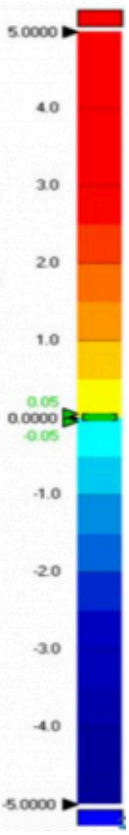

Fig. 5. Geometric Deviation (mm) of Pyramid with respect to CAD geometry; a) Blank as received, b) $15 \%$ Uniaxial pre-strained blank, c) Deep Drawn part.

\section{Conclusions}

This paper contributed to lay the ground for a better understanding of a novel circular economy strategy for metal recovery. Two different aspects of SPIF based Reshaping processes have been studied, namely, the change in formability as well as the shape accuracy performance of EoL components. To this aim process chains made of different prestraining levels followed by SPIF were replicated, strain path, $\alpha$ max and, final shape accuracy have been determined. Results revealed that SPIF, used for Reshaping, performed well for both the analyzed criteria. In fact, concerning the accuracy even an improvement was observed for a higher extent of deformation. Concerning the formability, although a slight reduction of the $\alpha$ max value was observed, the reshaping process can rely on satisfactory amount of available formability.

Next research step will concern a better understanding of the formability and accuracy change with varying both the Primary as well as the Reshaping process, for example, the analysis of Reshaping a biaxial pre-strained blank or forming a different geometry during the reshaping step.

\section{Bibliography}

[1] Worrell, E. Allwood, J. Gutowski, T. The Role of Material Efficiency in Environmental Stewardship. Annual Review of Environment and Resources, 2016, 41:575-98.

[2] Tolio, T. Bernard, A. Colledani, M. Kara, S. Seliger, G. Duflou, JR. Battaia, O. Takata, S. Design, management and control of demanufacturing and remanufacturing systems. CIRP Annals - Manufacturing Technology, 2017, 66:585-609.

[3] Cooper, DR. Allwood, JM. Reusing Steel and Aluminum Components at End of Product Life. Environmental Science 
and Technology, 2012, 46:10334-10340.

[4] Brosius, A. Hermes, M. Ben Khalif, N. Trompeter, M. Tekkaya, AE. Innovation by forming technology: motivation for research. International Journal of Material Forming, 2019, 2:29-38.

[5] Takano, H. Kitazawa, K. Goto, T. Incremental forming of nonuniform sheet metal: Possibility of cold recycling process of sheet metal waste. International Journal of Machine Tools and Manufacture, 2008, 48:477-482.

[6] Abu-Farha, FK. Khraisheh, MK. An integrated approach to the Superplastic Forming of lightweight alloys: towards sustainable manufacturing. International Journal of Sustainable Manufacturing, 2008, 1:18-40.

[7] Ingarao, G. Zaheer, O. Campanella, D. Fratini, L. Re-forming end-of-life components through single point incremental forming. Manufacturing Letters, 2020, 24:132-135.

[8] Ingarao, G. Zaheer, O. Fratini, L. Manufacturing processes as material and energy efficiency strategies enablers: the case of Single Point Incremental Forming to reshape End-of-Life metal components. CIRP Journal of Manufacturing Science and Technology, 2021, 32:145-153.

PDF automatically generated on 2021-05-20 17:34:00

Article url: https://popups.uliege.be/esaform21/index.php?id=4315

published by ULiège Library in Open Access under the terms and conditions of the CC-BY License (https://creativecommons.org/licenses/by/4.0) 